\title{
Multimorbidity, Depression, and Mortality in Primary Care: Randomized Clinical Trial of an Evidence-Based Depression Care Management Program on Mortality Risk
}

\author{
Joseph J. Gallo, MD, MPH', Seungyoung Hwang, MS, MSE' , Jin Hui Joo, MD², \\ Hillary R. Bogner, MD, MSCE ${ }^{3}$, Knashawn H. Morales, SCD, Martha L. Bruce, PhD, MPH ${ }^{5}$, and \\ Charles F. Reynolds III, $M D^{6}$
}

\begin{abstract}
'Department of Mental Health, Johns Hopkins University Bloomberg School of Public Health, Baltimore, MD, USA; '²epartment of Psychiatry and Behavioral Sciences, Johns Hopkins University School of Medicine, Baltimore, MD, USA; ${ }^{3}$ Department of Family Medicine and Community Health, University of Pennsylvania Health System, Philadelphia, PA, USA; ${ }^{4}$ Department of Biostatistics and Clinical Epidemiology, University of Pennsylvania Perelman School of Medicine, Philadelphia, PA, USA; ${ }^{5}$ Department of Psychiatry, Weill Cornell Medical College, White Plains, NY, USA; ${ }^{6}$ Department of Psychiatry, University of Pittsburgh School of Medicine, Pittsburgh, PA, USA.
\end{abstract}

BACKGROUND: Two-thirds of older adults have two or more medical conditions that often take precedence over depression in primary care.

OBJECTIVE: We evaluated whether evidence-based depression care management would improve the long-term mortality risk among older adults with increasing levels of medical comorbidity.

DESIGN: Longitudinal analyses of the practicerandomized Prevention of Suicide in Primary Care Elderly: Collaborative Trial (PROSPECT). Twenty primary care practices randomized to intervention or usual care.

PATIENTS: The sample included 1204 older primary care patients completing the Charlson Comorbidity Index (CCI) and other interview questions at baseline.

INTERVENTION: For 2 years, a depression care manager worked with primary care physicians to provide algorithmbased care for depression, offering psychotherapy, increasing the antidepressant dose if indicated, and monitoring symptoms, medication adverse effects, and treatment adherence. MAIN MEASURES: Depression status based on clinical interview, CCI to evaluate medical comorbidity, and vital status at 8 years (National Death Index).

KEY RESULTS: In the usual care condition, patients with the highest levels of medical comorbidity and depression were at increased risk of mortality over the course of the follow-up compared to depressed patients with minimal medical comorbidity [hazard ratio 3.02 (95\% CI, 1.32 to 8.72)]. In contrast, in intervention practices, patients with the highest level of medical comorbidity and depression compared to depressed patients with minimal medical comorbidity were not at significantly increased risk [hazard ratio 1.73 (95\% CI, 0.86 to 3.96)]. Nondepressed patients in intervention and usual care practices had similar mortality risk.

CONCLUSIONS: Depression management mitigated the combined effect of multimorbidity and depression on mortality. Depression management should be integral to optimal patient care, not a secondary focus.

Trial Registration: ClinicalTrials.gov identifier: NCTO0000367

Received July 10, 2015

Revised September 4, 2015

Accepted September 16, 2015

Published online October 2, 2015
J Gen Intern Med 31(4):380-6

DOI: $10.1007 / \mathrm{s} 11606-015-3524-\mathrm{y}$

(c) Society of General Internal Medicine 2015

\section{INTRODUCTION}

Older adults rarely present to the primary care doctor with a single medical condition. About two-thirds of Medicare beneficiaries have two or more medical conditions, and the prevalence of multimorbidity increases with advancing age. ${ }^{1}$ Multimorbidity, often defined as two or more concurrent medical conditions, has received attention as a focus of intervention because of its association with increased risk for all-cause mortality, functional impairment, and reduced quality of life. ${ }^{2}$ Multimorbidity poses significant challenges to clinicians, given the limited time and resources of primary care practice, and most guidance on appropriate care focuses on only one medical condition at a time. ${ }^{3}$

Should medical conditions such as diabetes and heart disease take precedence over depression, or should the management of depression be integral to the care of patients with these conditions? The co-occurrence of depression and medical multimorbidity can significantly complicate the management of older patients, yet most studies focus on one comorbidity at a time (e.g., diabetes and depression). Few studies are consistent with the multimorbidity that characterizes older adults in primary care. Depression often accompanies medical conditions such as hypertension, heart disease, diabetes, chronic lung disease, arthritis, and cancer. ${ }^{4}$ Among Medicare beneficiaries with a claim for depression, $90 \%$ have one or more associated chronic conditions. ${ }^{1}$ Patients in whom depression coexists with medical conditions may be less adherent to medical or behavioral regimens, have more functional impairment, and increased mortality. ${ }^{5}$ Compared to persons without depression or other psychiatric conditions, persons with comorbid psychiatric disorders and medical conditions such as diabetes and cardiovascular disease may receive a poorer quality of medical care. ${ }^{6}$ Observational studies have 
reported beneficial outcomes of depression treatment for patients with medical comorbidity, but no randomized trials have assessed how the treatment of depression may modify the risk of mortality among persons with multiple medical conditions.

In this investigation, we probe the relationship between multimorbidity and mortality among depressed older adults in primary care practices with a depression management program. Using the Prevention of Suicide in Primary Care Elderly: Collaborative Trial (PROSPECT) data, ${ }^{7}$ we hypothesized that medical comorbidity would be associated with allcause mortality over the 8-year study period regardless of intervention and depression status. Because PROSPECT tested a care management intervention to provide algorithmbased depression treatment against physician usual care, we also hypothesized that exposure to the intervention condition would attenuate the relationship between medical comorbidity and mortality among older persons with depression. In contrast to most randomized clinical trials, we followed patients who did not meet criteria for depression, providing a benchmark for gauging the influence of multimorbidity on mortality in practices with and without the depression management program. We have previously reported that the intervention was associated with reduction in mortality over the course of the follow-up, ${ }^{8}$ and we speculated that treating depression would reduce mortality risk among persons with multimorbidity to the level of mortality of persons with minimal levels of multimorbidity.

\section{METHODS}

\section{Study Sample}

The Prevention of Suicide in Primary Care Elderly: Collaborative Trial (PROSPECT) was a cluster-randomized, controlled trial comparing a primary care-based intervention with usual care to improve outcomes of depression. ${ }^{7,8}$ The study was conducted in 20 primary care practices in New York City, Philadelphia, and Pittsburgh from May 1999 to August 2001, with individual patients followed clinically for 2 years. Practices were randomized by coin flip to the intervention condition or to usual care after pairing by academic affiliation, size, setting, urban location, and population type. Patients aged 60 years and older who gave oral consent were screened for enrollment using the Centers for Epidemiologic Studies Depression scale $\left(\right.$ CES-D $\left.{ }^{9}\right)$. All patients who scored higher than 20 on the CES-D were invited into the study, as well as a $5 \%$ random sample of patients with lower scores. Approximately half of the final PROSPECT sample consisted of persons who met criteria for depression. Study procedures were implemented with written informed consent. The research protocols received full review and approval from the Institutional Review Boards of Cornell University, the University of Pennsylvania, and the University of Pittsburgh.

\section{Key Variables under Study}

Medical Comorbidity. Medical burden was assessed using the Charlson Comorbidity Index $\left(\mathrm{CCI}^{10}\right)$ and supplemental questions about the common disabling conditions of late life at baseline. The range of the scores for CCI is 0 to 12 , with higher scores indicating greater medical comorbidity burden. The CCI was developed with 19 medical categories to predict mortality in hospitalized patients. ${ }^{10}$ With both self-report data and medical claims coding, the CCI has been shown to be associated with service use, costs, and mortality in primary care. $^{11,12}$

Depression. Trained research assistants used the Diagnostic and Statistical Manual of Mental Disorders, 4th edition (DSMIV), to diagnose major depressive disorder (MDD). ${ }^{13}$ Clinically significant minor depression was defined as meeting DSM-IV criteria for minor depression with four depressive symptoms, Hamilton Depression Rating Scale (HDRS) score 10 or higher, and duration of symptoms 4 weeks or more. ${ }^{14}$ Structured Clinical Interview for Axis I DSM-IV Disorders (SCID) was obtained for all participants. ${ }^{15}$ Physicians received written notification of the depression status of patients based on the SCID assessment. For this analysis, we primarily considered depression as present if the criteria for either major or minor depression were met. Patients who did not meet criteria for depression provide a comparison to assess the impact of multimorbidity on mortality risk in patients from the same practices as depressed patients.

Vital Status. Vital status was determined using the National Death Index (NDI Plus), the computerized national death certificate registry of the National Center for Health Statistics. We did not transmit any study data along with identifying data or transmit identifying data through e-mail. The three PROSPECT sites verified vital status information obtained from NDI and sent the final version indexed by unique study identifier and stripped of personal identifiers to the University of Pennsylvania Data Core to produce the analytic data set. Written consent, including permission to obtain death certificate information, had been obtained from each participant. This study received approval from the Institutional Review Boards at Cornell University, the University of Pennsylvania, the University of Pittsburgh, and Johns Hopkins University, and independent review at the National Center for Health Statistics.

Other Covariates. Sociodemographic characteristics were assessed with standard questions regarding age, gender, selfidentified ethnicity, level of education attainment, and marital status. Smoking status was based on report of smoking within 6 months of the interview. Severity of depression was assessed using the 24-item Hamilton Depression Rating Scale (HDRS) ranging 0 to 76 with higher scores indicating greater depressive symptoms. ${ }^{14}$ The Mini-Mental State Examination (MMSE) was used to assess cognition. ${ }^{16}$ The range of scores 
for the MMSE is 0 to 30, with lower scores indicating greater cognitive impairment. Patients with scores less than 18 were not included in the study. The presence of suicidal ideation was assessed using the 21-item Scale for Suicidal Ideation (SSI) dichotomized to indicate the presence of any suicidal ideation. ${ }^{17}$

\section{Description of Usual Care and Intervention Conditions}

Practices randomized to Usual Care received notification of the depression status of their patients but no specific recommendations. Practices randomized to the Intervention Condition had available depression care managers (DCMs) who worked within the practice. DCMs implemented the intervention among patients with major or minor depression by working with primary care physicians to recommend treatment according to standard guidelines. DCMs followed up patients over 2 years to monitor treatment response, adherence, and side effects. First-line treatment was citalopram. Interpersonal psychotherapy could be used alone or as an augmentation. Patients in PROSPECT's intervention arm received acute and maintenance treatment over 2 years. In both study arms, physicians were informed by letter if patients reported any suicidal ideation and immediately when patients were identified at high suicide risk. Other sources detail the role of DCMs, pharmacotherapy strategy, and management of suicidal ideation. ${ }^{18-21}$

\section{Analytic Strategy}

Our analysis proceeded in two phases. First, to identify potential confounders of the relationship between medical comorbidity and mortality for use in adjusted models, we compared baseline sociodemographic, behavioral, and clinical characteristics of depressed and nondepressed patients across four quartiles of CCI scores. We sorted patients into four quartiles of Charlson scores due to the highly skewed distribution of scores and to facilitate comparisons across depression status and intervention condition. Bivariate comparisons for both continuous and binary characteristics were based on the $F$ value from linear and logistic regression models with random effects to account for clustering of patients by practice. Associations between baseline characteristics and time to death were assessed using Wald-type $\chi^{2}$ tests from Cox proportional hazard models. Characteristics that were both significantly different across quartiles of Charlson scores and associated with mortality at $\alpha=0.10$ significance level were considered potential confounders and included in adjusted models in subsequent phases of the analysis. ${ }^{22}$

In the second phase of the analysis, we used Cox proportional hazards regression to model the risk of medical comorbidity within depression and intervention strata, adjusting standard errors for within practice clustering. We carried out survival models treating the Charlson scores as continuous as well as in quartiles. We introduced a three-way interaction among medical comorbidity, baseline depression status (major and minor depression versus none), and intervention assignment into the Cox model in addition to main effects and corresponding two-way interactions, estimating hazard ratios and corresponding $95 \%$ confidence intervals stratified by depression and intervention status. Consistent with the literature, ${ }^{23}$ we set $\alpha$ at 0.10 to denote statistical significance for the three-way interaction term in the Cox proportional hazards model. There was no evidence of violation of the proportional hazards assumption, as the weighted Schoenfeld residuals were not associated with time. ${ }^{24} \mathrm{We}$ assessed for linearity between the CCI score and log hazard of mortality using the restricted cubic spline. ${ }^{25}$ We generated a display of the adjusted hazard of mortality with the Charlson comorbidity scores as a visual depiction of the functional association between CCI and mortality. Statistical analyses were performed using SAS, version 9.4 (SAS Institute Inc., Cary, NC).

\section{RESULTS}

\section{Sample Characteristics}

The CONSORT (Consolidated Standards for Reporting of Trials) flow diagram for PROSPECT has been published previously. ${ }^{7}$ In brief, the study screened 9072 patients aged 60 years and older, of whom 1888 were invited to participate, and $1238(65.8 \%)$ agreed to a baseline interview. Among them, we excluded 34 persons because of incomplete information on medical comorbidity, leaving a sample size of 1204 .

The mean CCI score of the entire sample was 2.6 (SD 2.3; median 2; interquartile range 1-4). At baseline, 583 people were classified as having major or clinically significant minor depression, and 621 did not meet criteria for depression. Overall, depressed patients had a significantly higher CCI score than nondepressed patients [mean (SD) 3.0 (2.4) versus 2.3 (2.1), respectively; $P<0.001]$. Table 1 compares the characteristics of depressed persons (major and minor) according to CCI quartiles, while Table 2 compares characteristics of persons who did not meet criteria for depression. In each table, the rightmost column provides the hazard ratio for mortality for each variable.

The mean years of schooling and MMSE scores for both depressed and nondepressed patients were inversely related to CCI scores. Baseline age, sex, education, depression severity (Hamilton Depression Rating Scale), cognition, and suicidal ideation met criteria for potential confounding variables as they differed significantly across CCI quartiles and were associated with mortality, and they were subsequently used in the multivariate models.

\section{Medical Comorbidity and Mortality}

After a median of 8.2 years of follow-up through 2008, 397 patients had died. Table 3 provides unadjusted and adjusted hazard ratio estimates representing the relationship between 
Table 1 Characteristics of the Depressed Study Sample*

\begin{tabular}{|c|c|c|c|c|c|c|c|}
\hline \multirow[t]{3}{*}{ Characteristics } & \multirow[t]{3}{*}{$\begin{array}{l}\text { Depressed } \\
(n=583)\end{array}$} & \multirow[t]{3}{*}{$\begin{array}{l}\text { CCI 0 } \\
(n=60)\end{array}$} & \multirow[t]{3}{*}{$\begin{array}{l}\text { CCI 1 } \\
(n=132)\end{array}$} & \multirow[t]{3}{*}{$\begin{array}{l}\text { CCI 2-3 } \\
(n=185)\end{array}$} & \multirow[t]{3}{*}{$\begin{array}{l}\text { CCI 4+ } \\
(n=206)\end{array}$} & \multirow{3}{*}{$\begin{array}{l}\text { Test of equality } \\
\text { Across groups, } \\
P \dagger\end{array}$} & \multirow{3}{*}{$\begin{array}{l}\begin{array}{l}\text { Association with } \\
\text { time to death }\end{array} \\
\text { Hazard ratio } \\
(95 \% \mathrm{CI}) \$\end{array}$} \\
\hline & & & & & & & \\
\hline & & & & & & & \\
\hline \multicolumn{8}{|l|}{ Sociodemographic } \\
\hline Mean (SD) age, years & $70.3(7.9)$ & $68.6(6.8)$ & $70.4(7.0)$ & $70.7(8.2)$ & $70.2(8.4)$ & 0.04 & $1.08(1.06-1.11)$ \\
\hline Females & $422(72.4)$ & $42(70.0)$ & $100(75.8)$ & $134(72.4)$ & $146(70.9)$ & 0.85 & $0.72(0.49-1.05)$ \\
\hline Ethnic minority & $193(33.1)$ & $17(28.3)$ & $44(33.3)$ & $48(25.9)$ & $84(40.8)$ & $<0.001$ & $0.89(0.62-1.27)$ \\
\hline Mean (SD) education, years & $12.7(3.3)$ & $13.9(3.7)$ & $13.0(3.0)$ & $12.7(3.0)$ & $12.1(3.4)$ & 0.02 & $0.98(0.94-1.02)$ \\
\hline Married & $215(36.9)$ & $21(35.0)$ & $57(43.2)$ & $68(36.8)$ & $69(33.5)$ & 0.32 & $0.74(0.54-1.01)$ \\
\hline \multicolumn{8}{|l|}{ Habits } \\
\hline Current smoker & $113(19.4)$ & $13(21.7)$ & $24(18.2)$ & $34(18.4)$ & $42(20.4)$ & 0.87 & $1.60(1.19-2.15)$ \\
\hline \multicolumn{8}{|l|}{ Psychiatric and cognitive status } \\
\hline HDRS score, mean (SD) & $18.1(6.0)$ & $16.6(4.9)$ & $16.4(5.1)$ & $18.5(6.6)$ & $19.3(5.9)$ & $<0.001$ & $1.00(0.98-1.02)$ \\
\hline MMSE score, mean (SD) & $27.5(2.5)$ & $28.1(1.7)$ & $28.0(1.8)$ & $27.4(2.5)$ & $26.9(2.8)$ & $<0.001$ & $0.91(0.85-0.97)$ \\
\hline Suicidal ideation & $145(24.9)$ & $9(15.0)$ & $30(22.7)$ & $48(25.9)$ & $58(28.2)$ & 0.049 & $1.07(0.85-1.35)$ \\
\hline \multicolumn{8}{|l|}{ Treatment assignment } \\
\hline Intervention practice & $310(53.2)$ & $30(50.0)$ & $74(56.1)$ & $97(52.4)$ & $109(52.9)$ & $<0.001$ & $0.99(0.76-1.30)$ \\
\hline
\end{tabular}

$C I=$ Confidence interval; $S D=$ standard deviation; $H D R S=$ Hamilton Depression Rating Scale; MMSE=Mini-Mental State Examination

* Data are numbers (percentages) unless otherwise indicated

${ }^{\prime} F$ test from linear and logistic regression with random effects

${ }^{*} \chi^{2}(d f=1) 95 \%$ confidence intervals based on bivariate Cox proportional hazards model

CCI scores and 8-year mortality, stratified by both patient baseline depression status and practice intervention group. We observed no significant departure from linearity between the CCI and $\log$ hazard of mortality $\left(P=0.46 ; \chi^{2}=0.54, \mathrm{df}=1\right)$. Higher CCI scores were associated with a greater risk of death, regardless of intervention or depression status (adjusted hazard ratios per 1-unit increase of CCI ranging from 1.12 to 1.25 in Table 3).

\section{Medical Comorbidity and Mortality and the Depression Management Program}

Depressed patients (top half of Table 3 ) in the highest quartile of the CCI (having a score of 4 or more) in usual care were more likely to die than were persons in the lowest quartile [having a score of 0 ; hazard ratio $3.02(95 \% \mathrm{CI}, 1.32$ to 8.72$)]$. In contrast, in the intervention practices, depressed patients in the highest quartile of the $\mathrm{CCI}$ were at no greater risk than were persons in the lowest quartile [hazard ratio 1.73 ( $95 \% \mathrm{CI}, 0.86$ to 3.96)]. Nondepressed patients provided a benchmark for interpreting the effect of the depression management program on depressed patients. Compared to persons without depression in usual care practices, persons without depression in the intervention practices had a similar risk of mortality whether examined as a continuous variable or in quartiles (lower half of Table 3). According to our pre-specified $\alpha$ level of significance for a three-way interaction, exposure to the intervention reduced the risk of mortality at higher levels of medical comorbidity $\left(P=0.07 ; \chi^{2}=3.26\right.$, $\left.\mathrm{df}=1\right)$.

Table 2 Characteristics of the Nondepressed Study Sample*

\begin{tabular}{|c|c|c|c|c|c|c|c|}
\hline \multirow[t]{3}{*}{ Characteristics } & \multirow[t]{3}{*}{$\begin{array}{l}\text { Nondepressed } \\
(n=621)\end{array}$} & \multirow[t]{3}{*}{$\begin{array}{l}\text { CCI 0 } \\
(n=111)\end{array}$} & \multirow[t]{3}{*}{$\begin{array}{l}\text { CCI 1 } \\
(n=160)\end{array}$} & \multirow[t]{3}{*}{$\begin{array}{l}\text { CCI 2-3 } \\
(n=209)\end{array}$} & \multirow[t]{3}{*}{$\begin{array}{l}\text { CCI 4+ } \\
(n=141)\end{array}$} & \multirow{3}{*}{$\begin{array}{l}\text { Test of equality } \\
\text { Across groups, } \\
P_{\dagger}\end{array}$} & \multirow{3}{*}{$\begin{array}{l}\begin{array}{l}\text { Association with } \\
\text { time to death }\end{array} \\
\text { Hazard ratio } \\
(95 \% \mathrm{CI}) \$\end{array}$} \\
\hline & & & & & & & \\
\hline & & & & & & & \\
\hline \multicolumn{8}{|l|}{ Sociodemographic } \\
\hline Mean (SD) age, years & $71.8(7.7)$ & $70.5(7.3)$ & $71.9(8.0)$ & $72.1(7.9)$ & $72.4(7.3)$ & 0.26 & $1.07(1.06-1.09)$ \\
\hline Females & $422(68.0)$ & $70(63.1)$ & $117(73.1)$ & $141(67.5)$ & $94(66.7)$ & 0.01 & $0.53(0.38-0.74)$ \\
\hline Ethnic minority & $204(32.9)$ & $29(26.1)$ & $41(25.6)$ & $64(30.6)$ & $70(49.6)$ & $<0.001$ & $0.86(0.64-1.15)$ \\
\hline Mean (SD) education, years & $13.2(3.8)$ & $14.3(3.6)$ & $13.4(3.7)$ & $13.0(3.8)$ & $12.2(3.8)$ & $<0.001$ & $0.94(0.89-0.98)$ \\
\hline Married & $248(39.9)$ & $56(50.5)$ & $73(45.6)$ & $68(32.5)$ & $51(36.2)$ & $<0.001$ & $0.86(0.65-1.15)$ \\
\hline \multicolumn{8}{|l|}{ Habits } \\
\hline Current smoker & 72 (11.6) & $12(10.8)$ & $16(10.0)$ & $29(13.9)$ & 15 (10.6) & 0.35 & $1.84(1.35-2.49)$ \\
\hline Psychiatric and cognitive status & & & & & & & \\
\hline HDRS score, mean (SD) & $5.2(4.2)$ & $4.4(4.3)$ & $4.7(3.7)$ & $5.5(4.3)$ & $5.9(4.3)$ & 0.027 & $1.03(1.01-1.06)$ \\
\hline MMSE score, mean (SD) & $27.5(2.4)$ & $28.4(1.6)$ & $27.9(2.0)$ & $27.1(2.7)$ & $27.0(2.8)$ & $<0.001$ & $0.88(0.84-0.93)$ \\
\hline Suicidal ideation $(\mathrm{SSI}>0)$ & $46(7.4)$ & $8(7.2)$ & $10(6.3)$ & $21(10.0)$ & $7(5.0)$ & 0.092 & $1.71(1.25-2.36)$ \\
\hline \multicolumn{8}{|l|}{ Treatment assignment } \\
\hline Intervention practice & $285(45.9)$ & $52(46.8)$ & $79(49.4)$ & 89 (42.6) & $65(46.1)$ & $<0.001$ & $1.22(0.97-1.54)$ \\
\hline
\end{tabular}

$C I=$ Confidence interval; $S D=$ standard deviation; HDRS=Hamilton Depression Rating Scale; MMSE=Mini-Mental State Examination

* Data are numbers (percentages) unless otherwise indicated

${ }^{\dagger} F$ test from linear and logistic regression with random effects.

${ }^{*} \chi^{2}(d f=1) 95 \%$ confidence intervals based on bivariate Cox proportional hazards model 
Table 3 Relationship of Baseline Comorbidity and All-cause Mortality, by Depression Status and Practice Intervention Group*

\begin{tabular}{|c|c|c|c|c|c|}
\hline \multirow[t]{3}{*}{ Depression status and CCI } & \multirow[t]{3}{*}{$\mathbf{N}$} & \multicolumn{2}{|c|}{ Intervention practices } & \multicolumn{2}{|c|}{ Usual care practices } \\
\hline & & Unadjusted HR & Adjusted HR $\dagger$ & Unadjusted HR & Adjusted HR \\
\hline & & $(95 \% \mathrm{CI})$ & $(95 \% \mathrm{CI})$ & $(95 \% \mathrm{CI})$ & $(95 \% \mathrm{CI})$ \\
\hline \multicolumn{6}{|l|}{ All depressed patients } \\
\hline Continuous & 583 & $1.16(1.08-1.24)$ & $1.12(1.04-1.20)$ & $1.24(1.15-1.34)$ & $1.25(1.14-1.35)$ \\
\hline \multicolumn{6}{|l|}{ Categorical } \\
\hline CCI 0 & 60 & 1.00 & 1.00 & 1.00 & 1.00 \\
\hline CCI 1 & 132 & $1.18(0.55-2.81)$ & $1.18(0.55-2.81)$ & $1.28(0.48-4.03)$ & $1.09(0.40-3.43)$ \\
\hline CCI 2-3 & 185 & $1.19(0.57-2.77)$ & $0.95(0.46-2.22)$ & $2.23(0.94-6.57)$ & $1.93(0.82-5.70)$ \\
\hline CCI $4+$ & 206 & $2.03(1.02-4.63)$ & $1.73(0.86-3.96)$ & $4.04(1.78-11.62)$ & $3.02(1.32-8.72)$ \\
\hline \multicolumn{6}{|l|}{ Nondepressed patients } \\
\hline Continuous & 621 & $1.11(1.02-1.21)$ & $1.13(1.03-1.23)$ & $1.15(1.05-1.25)$ & $1.12(1.02-1.23)$ \\
\hline \multicolumn{6}{|l|}{ Categorical } \\
\hline CCI 0 & 111 & 1.00 & 1.00 & 1.00 & 1.00 \\
\hline CCI 1 & 160 & $1.08(0.54-2.26)$ & $0.76(0.38-1.60)$ & $0.91(0.44-1.90)$ & $0.88(0.42-1.88)$ \\
\hline CCI 2-3 & 209 & $1.41(0.74-2.85)$ & $1.05(0.55-2.13)$ & $1.37(0.74-2.69)$ & $1.16(0.63-2.29)$ \\
\hline CCI $4+$ & 141 & $2.17(1.14-4.40)$ & $1.84(0.96-3.76)$ & $2.00(1.06-3.96)$ & $1.59(0.84-3.17)$ \\
\hline
\end{tabular}

$C C I=$ Charlson Comorbidity Index; HR=hazard ratio; $C I=$ confidence interval

* Hazard ratios are from Cox proportional hazards models

${ }^{*}$ Adjusted models include terms for baseline age, sex, education, depression severity, cognition, and suicidal ideation

The conclusions are not changed if only the 158 persons with major depression and the highest level of medical comorbidity were examined. In usual care, among persons with the highest level of medical comorbidity and major depression, the risk of death was three-fold higher than for persons with similar medical comorbidity who did not meet criteria for depression [adjusted hazard ratio 3.17 (95\% CI, 1.26 to 10.44)]. In intervention practices, patients with major depression and the highest level of medical comorbidity were not statistically significantly at increased risk [adjusted hazard ratio 2.76 (95\% CI, 0.97 to 10.94$)]$.

Figure 1 illustrates that as the CCI score increases, so does the relative risk of death (with a CCI score of zero as the comparison group at each level of multimorbidity). Among nondepressed groups, the risk relationship of multimorbidity to mortality is similar in both usual care and intervention practices. However, the risk of death increases with increasing multimorbidity only for the depressed patients in usual care, not in intervention practices.

\section{DISCUSSION}

We found that multimorbidity associated with depression increased mortality among older primary care patients, but that a depression management program could mitigate the combined effect of multimorbidity and depression on mortality. We probed medical comorbidity as a construct based on the Charlson Comorbidity Index, a well-studied, widely used standard method of assessing medical comorbidity in use for almost 30 years. Whether among depressed or nondepressed patients, the higher comorbidity was associated with increased risk of death. Among older adults with the most medical comorbidity, depressed patients in usual care were at increased risk of death, while depressed patients in intervention practices were not. Nondepressed patients provided a benchmark, since the mortality experience of nondepressed patients as a function of increasing comorbidity was the same in intervention and usual care practices. Our focus on multimorbidity here extends our previous findings that the intervention was associated with decreased mortality over the course of the follow-up. ${ }^{8}$ Sustained depression management over at least 2 years can improve mortality even in the face of multimorbidity.

Before discussing the implications of our results, limitations should be acknowledged. Participating primary care practices

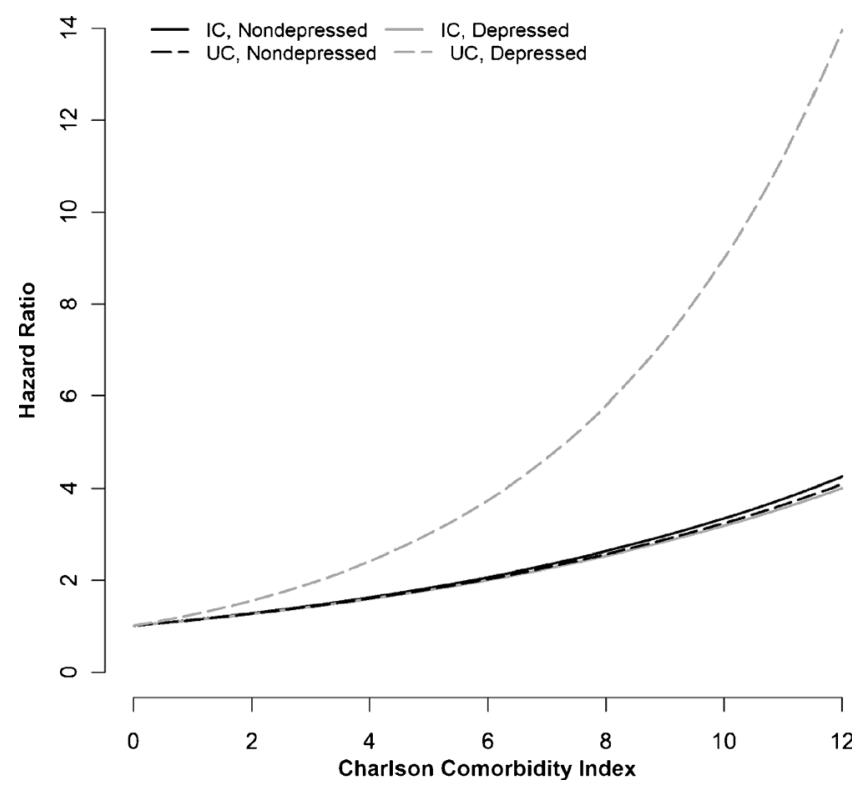

Fig. 1 Adjusted relative hazard of all-cause mortality. Relative hazard predicted using Cox proportional hazards regression for depressed (gray) and nondepressed (black) patients in intervention practices (solid) or usual care practices (dashed), adjusted for baseline age, sex, education, depression severity, cognition, and suicidal ideation. The curves show adjusted hazard ratios (HRs). A CCI of zero was used as the reference $(\mathrm{HR}=1)$ 
may not reflect all primary care practices in the US, because the study was carried out at sites in the greater metropolitan areas of New York City, Philadelphia, and Pittsburgh. The diagnosis of chronic medical conditions was based on patient interviews. However, self-reported data on chronic diseases are meaningful and reliable. ${ }^{26}$ The mortality reduction among patients with depression and high burden of medical comorbidities randomized to the intervention may be due to factors other than the specific effects of a depression management program. We note that the similar relationship of multimorbidity to mortality among nondepressed patients in usual care and intervention practices suggests that the differences we observed among depressed patients were not due to the case mix, physician practice patterns, or other unmeasured characteristics of the practices. Misclassification of vital status was also a potential limitation. However, overall sensitivity of the NDI for ascertainment of vital status has generally been well over $90 \%$ in most studies. ${ }^{27}$ The Charlson Comorbidity Index is based on a list of medical conditions and does not include geriatric syndromes such as falls or urinary incontinence that may contribute to poor quality of life and mortality.

We believe that our findings deserve attention because addressing mental disorders such as depression often takes a back seat in the face of significant medical comorbidity (the "competing demands" of primary care practice ${ }^{28}$ ). In contrast, we found that multimorbidity was no barrier to response to a sustained intervention consisting of depression care management in primary care. The paradox is that physicians may be more aware of depression in the context of medical comorbidity, but recognition does not necessarily mean attention to treating depression. For example, Bogner and colleagues found that, compared to patients without heart disease, patients with heart disease were more likely to be identified as depressed by the primary care physician, but less likely to be actively managed for depression. ${ }^{29}$ Koike and colleagues found that rates of depression treatment in patients with medical disorders who participated in a quality improvement program were low despite the greater severity of depression. ${ }^{30}$ In summary, such studies demonstrate patterns of practice that downplay the importance of treating depression.

Observational evidence supports the benefits of depression treatment in those with medical illness. A Cochrane collaboration meta-analysis concluded that efficacy of antidepressants in the medically ill was similar to that in patients without medical comorbidity. ${ }^{31}$ Simon and colleagues observed that persons who started antidepressant treatment in primary care experienced significant improvement in depression as well as function and disability. ${ }^{32}$ The reduction in disability following depression treatment was as great in those with multimorbidity as in those without medical conditions. Although antidepressants are effective for treating depression in patients with a wide range of physical diseases, antidepressants do not reliably affect disease self-management behaviors in chronically ill patients, ${ }^{31}$ which suggests the potential benefits of educational and psychosocial treatments. Psychosocial treatments such as medical condition-specific education may improve depression and anxiety. ${ }^{33}$ Evidence-based depression care management improves glycemic control in patients with cooccurring diabetes and depression. ${ }^{34}$ Sustained management may be essential to achieving improved health goals, since participants in PROSPECT received acute and maintenance treatment over 2 years, both pharmacologic and nonpharmacologic. ${ }^{7}$

In our study, primary care older adults with more comorbidities in the intervention condition benefited in terms of mortality. Though this may seem counterintuitive, other studies have shown that sicker patients may have more to gain with appropriate intervention. Harrison and colleagues showed that patients with multiple physical conditions and depression who received chronic disease management support improved considerably more compared to patients with fewer medical problems or those without depression. ${ }^{35}$ Although depression is often associated with reduced self-management in observational studies, ${ }^{36}$ patients with medical conditions and depression demonstrate greater benefits after receiving chronic disease management support. ${ }^{35,37}$ In combination with our study, the evidence suggests that depression management should not be secondary to management of 'organic' disease but rather should be integrated into the care of older adults with multimorbidity.

Acknowledgments: This work was supported by grants from the National Institute of Mental Health (JG: R01 MH065539, K24 MH070407; JJ: K23 MH100705; HB: R21 MH094940, R34 MHO85880; KM: KO1 MHO73903; MB: P3O MH085943; CR: P3O MH090333).

Conflict of interest: No support from any organization for the submitted work; no financial relationships with any organizations that might have an interest in the submitted work in the previous 3 years; no other relationships or activities that could appear to have influenced the submitted work.

Corresponding Author: Joseph J. Gallo, MD, MPH; Department of Mental HealthJohns Hopkins University Bloomberg School of Public Health, 624 North Broadway, Baltimore, MD 21205, USA (e-mail: jgallo2@jhu.edu).

\section{REFERENCES}

1. Salive M. Multimorbidity in older adults. Epidemiol Rev. 2013;35:75-83.

2. Smith SM, Soubhi H, Fortin M, Hudon C, O'Dowd T. Interventions for improving outcomes in patients with multimorbidity in primary care and community settings. Cochrane Database Syst Rev. 2012;4:CD006560.

3. Boyd CM, Darer J, Boult C, Fried LP, Boult L, Wu AW. Clinical practice guidelines and quality of care for older patients with multiple comorbid diseases: implications for pay for performance. JAMA:J Am Med Assoc. 2005;294(6):716-24.

4. Sinnige $J$, Braspenning $J$, Schellevis F, Stirbu-Wagner I, Westert G, Korevaar J. The prevalence of disease clusters in older adults with multiple chronic diseases-a systematic literature review. PLoS One. 2013;8:11.

5. Mezuk B, Gallo JJ. Depression and medical illness in late life: Race, resources, and stress. In: Lavretsky $\mathrm{H}$, Sajatovic M, Reynolds CF, eds. Depression in Late Life. London: Oxford University Press; 2013:270-94.

6. Mitchell AJ, Malone D, Doebbeling CC. Quality of medical care for people with and without comorbid mental illness and substance misuse: systematic review of comparative studies. Br J Psychiatry. 2009;194(6):491-9. 
7. Bruce ML, Ten Have TR, Reynolds CF 3rd, et al. Reducing suicidal ideation and depressive symptoms in depressed older primary care patients: a randomized controlled trial. JAMA : J Am Med Assoc. 2004;291(9): 1081-91.

8. Gallo JJ, Morales KH, Bogner HR, et al. Long term effect of depression care management on mortality in older adults: follow-up of cluster randomized clinical trial in primary care. BMJ. 2013;346:f2570.

9. Charlson ME, Pompei P, Ales KL, Mackenzie CR. A new method of classifying prognostic comorbidity in longitudinal studies: development and validation. J Chron Dis. 1987;40(5):373-83.

10. Perkins AJ, Kroenke $\mathbf{K}$, Unutzer $\mathbf{J}$, et al. Common comorbidity scales were similar in their ability to predict health care costs and mortality. J Clin Epidemiol. 2004;57(10): 1040-8.

11. Huntley AL, Johnson R, Purdy S, Valderas JM, Salisbury C. Measures of multimorbidity and morbidity burden for use in primary care and community settings: a systematic review and guide. Ann Fam Med. 2012;10(2):134-41.

12. American Psychiatric Association. Diagnostic and Statistical Manual of Mental Disorders, DSM-IV. 4 ed: American Psychiatric Association; 1994.

13. Hamilton M. A rating scale for depression. J Neurol Neurosurg Psychiatry. 1960;23:56-62.

14. Spitzer R, Gibbon M, Williams J. Structured clinical interview for Axis I DSM-IV disorders (SCID). Washington, DC: American Psychiatric Association Press; 1995.

15. Folstein MF, Folstein SE, McHugh PR. "Mini-mental state". A practical method for grading the cognitive state of patients for the clinician. J Psychiatr Res. 1975;12(3):189-98.

16. Beck AT, Brown GK, Steer RA. Psychometric characteristics of the Scale for Suicide Ideation with psychiatric outpatients. Behav Res Ther. 1997;35(11):1039-46.

17. Brown GK, Bruce ML, Pearson JL. High-risk management guidelines for elderly suicidal patients in primary care settings. Int $J$ Geriatr Psychiatry. 2001;16(6):593-601.

18. Mulsant BH, Alexopoulos GS, Reynolds CF 3rd, et al. Pharmacological treatment of depression in older primary care patients: the PROSPECT algorithm. Int J Geriatr Psychiatry. 2001;16(6):585-92.

19. Reynolds CF, Degenholtz H, Parker LS, et al. Treatment as usual (TAU) control practices in the PROSPECT Study: managing the interaction and tension between research design and ethics. Int $\mathrm{J}$ Geriatr Psychiatry. 2001;16(6):602-8.

20. Schulberg HC, Bryce C, Chism K, et al. Managing late-life depression in primary care practice: a case study of the Health Specialist's role. Int $\mathrm{J}$ Geriatr Psychiatry. 2001;16(6):577-84.

21. Rothman KJ, Greenland S, Lash TL. Modern Epidemiology. 3rd ed. Philadelphia, PA: Lippincott, Williams, \& Wilkins; 2012.

22. Fleiss JL. Analysis of data from multiclinic trials. Control Clin Trials. 1986;7(4):267-75.
23. Allison PD. Survival analysis using SAS: A practical guide. 2nd ed. Cary, NC: SAS Institute; 2010.

24. Harrell FE. Regression Modeling Strategies With Applications to Linear Models, Logistic Regression, and Survival Analysis. New York, NY: Springer; 2001.

25. Bowlin SJ, Morrill BD, Nafziger AN, Lewis C, Pearson TA. Reliability and changes in validity of self-reported cardiovascular disease risk factors using dual response: the behavioral risk factor survey. J Clin Epidemiol. 1996;49(5):511-7.

26. Sathiakumar N, Delzell E, Abdalla O. Using the National Death Index to obtain underlying cause of death codes. J Occup Environ Med/Am Coll Occup Environ Med. 1998;40(9):808-13.

27. Nutting PA, Rost K, Smith J, Werner JJ, Elliot C. Competing demands from physical problems: effect on initiating and completing depression care over 6 months. Arch Fam Med. 2000;9(10):1059-64.

28. Bogner HR, Ford DE, Gallo JJ. The role of cardiovascular disease in the identification and management of depression by primary care physicians. Am J Geriatr Psychiatr. 2006;14(1):71-8.

29. Koike AK, Unutzer J, Wells KB. Improving the care for depression in patients with comorbid medical illness. Am J Psychiatry. 2002;159(10):1738-45.

30. Gill D, Hatcher S. A systematic review of the treatment of depression with antidepressant drugs in patients who also have a physical illness. J Psychosom Res. 1999;47(2):131-43.

31. Simon GE, Von Korff M, Lin E. Clinical and functional outcomes of depression treatment in patients with and without chronic medical illness. Psychol Med. 2005;35(2):271-9.

32. Kunik ME, Veazey C, Cully JA, et al. COPD education and cognitive behavioral therapy group treatment for clinically significant symptoms of depression and anxiety in COPD patients: a randomized controlled trial. Psychol Med. 2008;38(3):385-96.

33. Katon WJ, Lin EH, Von Korff $\mathbf{M}$, et al. Collaborative care for patients with depression and chronic illnesses. N Engl J Med. 2010;363(27):2611-20.

34. Harrison M, Reeves D, Harkness E, et al. A secondary analysis of the moderating effects of depression and multimorbidity on the effectiveness of a chronic disease self-management programme. Patient Educ Couns. 2012;87(1):67-73.

35. McKellar JD, Humphreys K, Piette JD. Depression increases diabetes symptoms by complicating patients' self-care adherence. Diabetes Educ. 2004;30(3):485-92.

36. Jerant A, Kravitz R, Moore-Hill M, Franks P. Depressive symptoms moderated the effect of chronic illness self-management training on selfefficacy. Med Care. 2008;46(5):523-531.

37. Radloff LS. The CES-D Scale: A self-report depression scale for research in the general population. Appl Psych Meas. 1977;1:385-401. 